\title{
9 TAgUngSDOKUMENTATION: SCHLÜSSELAKTEURE DER REgiONALENTWICKLUNG: Welche Perspektiven bietet Entrepre- NEURSHIP FÜR LÄNDLICHE RÄUME?
}

\section{Stefanie DöRINGER und Jakob EDER}

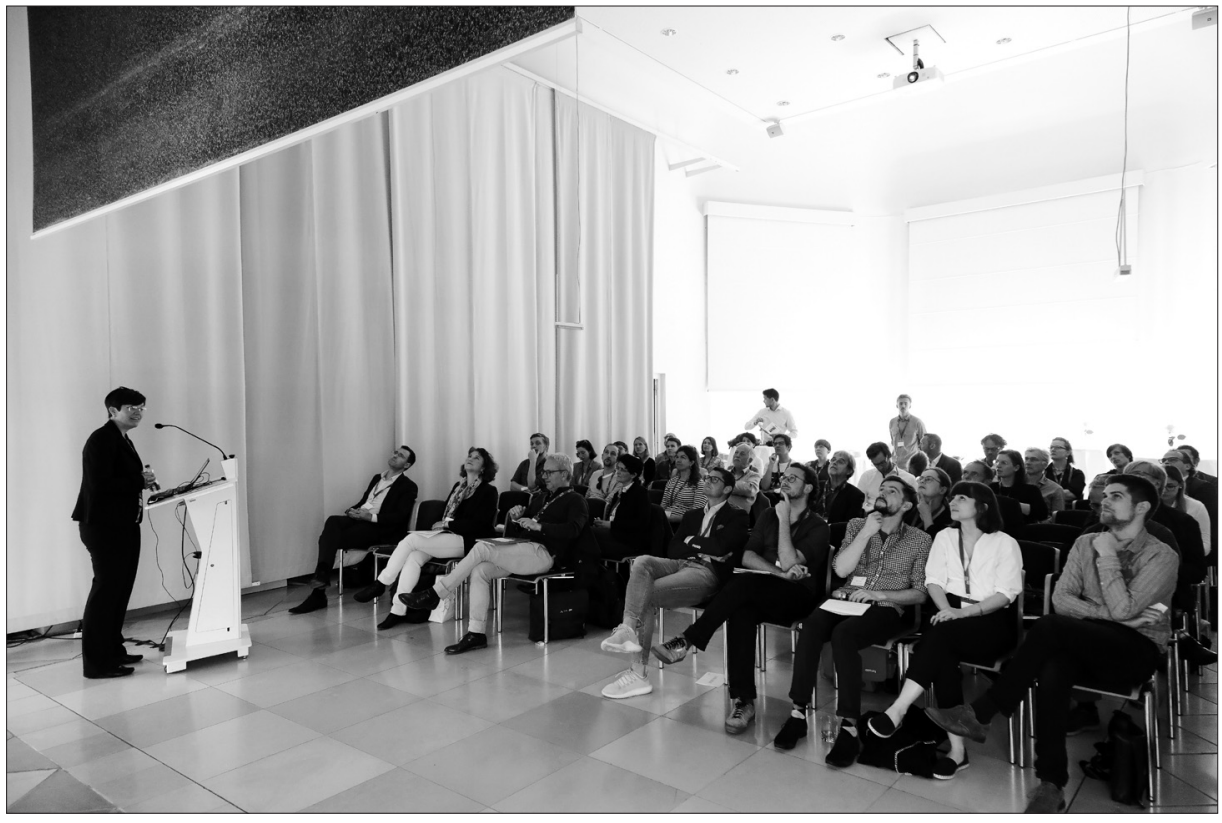

Bild 1: Key-Note-Vortragende Heike MAYER und Tagungsbesucherinnen und -besucher (Foto: C. Fürthner)

Viele ländliche Regionen sind derzeit mit ungünstigen Entwicklungen konfrontiert. Beispielsweise resultiert der demographische Wandel in einer alternden Bevölkerung und junge Menschen finden oft keine geeigneten Ausbildungs- oder Arbeitsplätze vor Ort. Diese Trends haben sich in den letzten Jahren verschärft, was die Regional- 
entwicklung vor zusätzliche Herausforderungen stellt. Vor diesem Hintergrund diskutierten ca. 50 Vertreterinnen und Vertreter aus Wissenschaft und Praxis auf Einladung des Instituts für Stadt- und Regionalforschung der Österreichischen Akademie der Wissenschaften, welche Impulse von Schlüsselakteuren in diesen Regionen ausgehen können. Die Tagung fand am 24. und 25. Mai 2019 in Wien statt.

Grundlage für die Tagung war der Begriff des „Entrepreneurship“, der bewusst breit ausgelegt war. Entrepreneure gründen einerseits Unternehmen, schaffen Arbeitsplätze und tragen so zu einer positiven Regionalentwicklung bei. Sie können aber auch gezielt auf Institutionen einwirken, ein Bewusstsein für relevante Themen schaffen und Netzwerke bilden, um steuernd einzugreifen. Schlüsselakteure können also aus Wirtschaft, Verwaltung, Politik oder Zivilgesellschaft stammen und dabei sowohl Individuen als auch Organisationen oder Vereine umfassen.

Der erste Tag stand ganz im Zeichen des Austauschs von Wissenschaft und Praxis. Die Vortragenden und das Publikum brachten dabei sowohl die wissenschaftliche als auch die praktische Erfahrung ein und sorgten so für eine lebendige Diskussion. Am zweiten Tag fand daran anknüpfend ein vertiefender wissenschaftlicher Workshop mit Inputs aus Deutschland, Österreich und der Schweiz statt.

In den beiden Keynotes des ersten Tages wurde die Vielfalt von Entrepreneurship diskutiert. Prof. Heike MAYER vom Geographischen Institut der Universität Bern stellte ihre Forschung der letzten Jahre zu innovativen Unternehmensgründungen in der Schweiz und im Alpenraum vor. Dabei ging sie auf die Unterschiede von Innovation in solchen Regionen im Vergleich zu Städten ein. Unternehmen greifen etwa seltener auf neueste Grundlagenforschung zurück, schätzen aber die hohe Loyalität ihrer Belegschaft und engagieren sich oft regional. Prof. Birgit LEICK vom Østfold University College erweiterte die unternehmerische Perspektive um das Konzept des institutionellen Entrepreneurs. Anhand von vier Fallbeispielen konnte sie zeigen, wer diese institutionellen Schlüsselakteure sind, was sie auszeichnet, aus welchen Gründen sie sich engagieren und wann sie aktiv werden.

Anschließend hielt Martin GrafFenBERGER vom Leibniz-Institut für Länderkunde einen angewandten Impulsvortrag zu aktueller empirischen Forschung zu Schlüsselakteuren in ländlichen Regionen. Mit empirischen Beispielen veranschaulichte er, dass diese in diesem Kontext besonders wichtig sind und entscheidende Impulse setzen, aber auch überbeansprucht werden können. Zwei Vorträge aus der Praxis bildeten den Schlusspunkt des ersten Tages. Patrick MüLLER, ein Unternehmer aus einer ländlichen, vom Tourismus geprägten Region, erzählte von den Schwierigkeiten, ein innovatives Unternehmen unter diesen Umständen aufzubauen, und wieso er es dennoch jederzeit wieder machen würde. Der Raumplaner Josef LuEGER zeigte schließlich, wie Schlüsselakteure aus Politik, Wirtschaft und Planung zusammengearbeitet haben, um notwendige Transformationsprozesse in einer niederösterreichischen Kleinstadt voranzutreiben. 
Im Rahmen des wissenschaftlichen Workshops am zweiten Tag wurden verschiedene Konzepte und Fallstudien zu Schlüsselakteuren in ländlichen Räumen diskutiert. Eröffnet wurde der erste Vortragsblock mit zwei Vorträgen zu Konzepten des policy und governance entrepreneurship. Während sich der erste Beitrag den Schlüsselakteuren der Regionalentwicklung in der Schweiz widmete (Yasmine WILLI), fokussierte der zweite Vortrag auf das Zusammenspiel von Akteuren aus Politik und Wirtschaft in österreichischen Kleinstädten (Stefanie DöRINGER). Im Anschluss daran thematisierte ein Beitrag aus Österreich die spezifische Rolle von Schlüsselakteuren in der regionalen Energietransformation vor dem Hintergrund des bricolage-Ansatzes (Martha ECKER). Der vierte Beitrag ermöglichte einen Einblick in das Handeln von Entrepreneuren in Innovations-Regimen in Ostdeutschland (Theresa MARTENS). Der zweite Vortragsblock fokussierte auf Entrepreneure aus dem Bereich der Wirtschaft. In diesem Kontext wurde eine empirische Studie zu „Rural Entrepreneuren“ und Unternehmensgründungen in Schweizer Bergregionen vorgestellt (Rahel MeILI). Der anschließende Vortrag wechselte die Perspektive und fokussierte auf das Engagement und die Verantwortung von Unternehmern in der Stadt- und Regionalentwicklung (Hans-Hermann ALBERS). Den Abschluss bildete ein empirischer Beitrag zu „Lifestyle Entrepreneuren“, die mit der Gründung von innovativen Kleinstunternehmen neue Wege im Norden Deutschlands beschreiten (Gesine TuITJER). Abgerundet wurde der Vormittag mit zwei Posterpräsentationen zu Schlüsselakteuren im Kontext von sozialen Innovationen in der Schweiz (Samuel WIRTH) und im Kontext der Pfadentwicklung in ehemals industriell geprägten Regionen in Deutschland (Franziska GörmaR).

Der intensive Austausch zwischen Wissenschaft und Praxis im Rahmen der Tagung hat gezeigt, dass Forschung in diesem Gebiet gesellschaftlich relevant ist und sich Schlüsselakteure in den Konzepten wiedererkennen. Vertreterinnen und Vertreter aus beiden Bereichen betonten folglich auch, dass die Wissenschaft hier die Nähe zur Praxis braucht und die Distanz geringer ist, als häufig angenommen. Die Fokussierung der Tagung auf eine überschaubare Thematik war dabei ein Vorteil, um inhaltlich in die Tiefe gehen zu können und um die zentralen Fragestellungen intensiv zu diskutieren. In diesem Sinne bot die Veranstaltung auch viele Einblicke, unter welchen Rahmenbedingungen Entrepreneurship in ländlichen Regionen stattfindet. Allerdings wurde auch die Notwendigkeit für weitere Forschung deutlich: Die Formulierung von konkreten Handlungsempfehlungen für die Regionalpolitik stellt eine große Herausforderung dar und erfordert eine weitere Vertiefung. Ein neues Forschungsfeld könnte auch darin begründet sein, verschiedene Ebenen von Entrepreneurship - sowohl unternehmerisch als auch institutionell - vergleichend in verschiedenen ländlichen Regionen zu analysieren. 


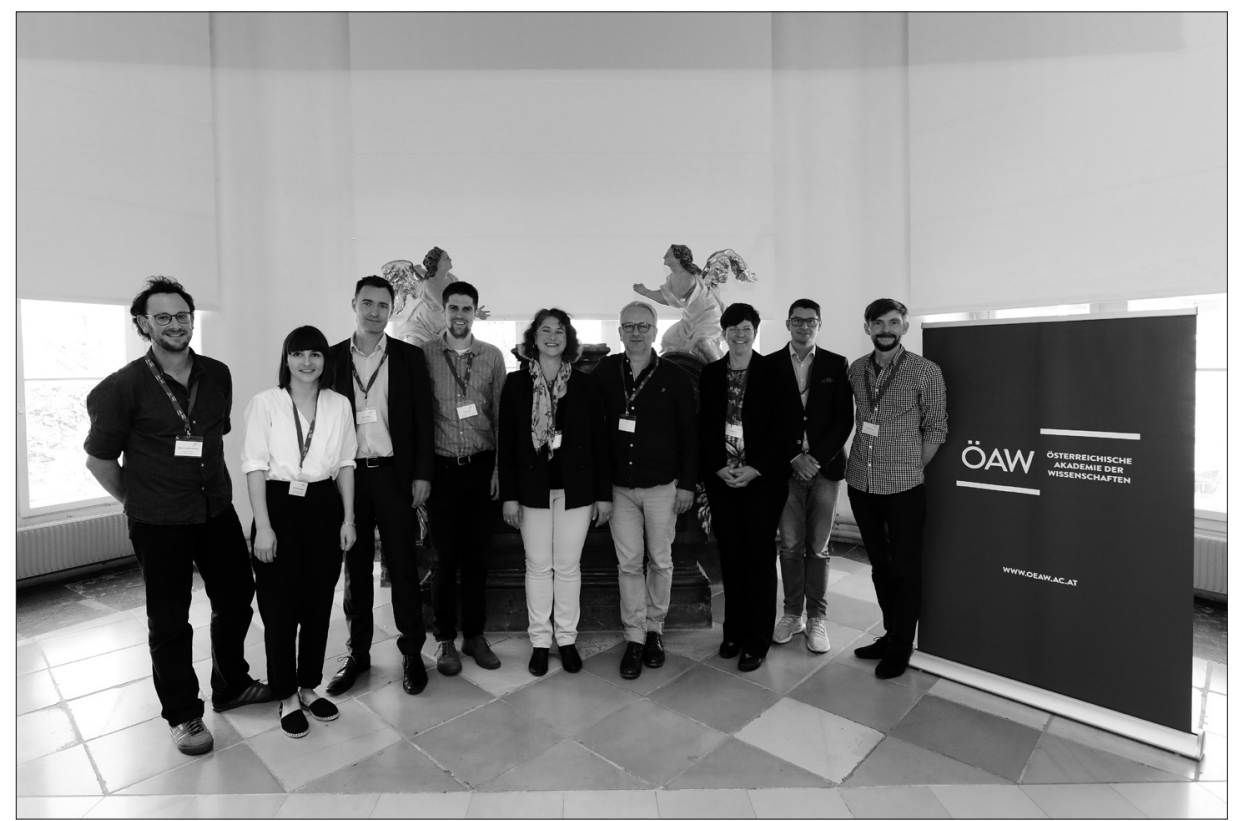

Bild 2: Vortragende und Organisatorenteam der Tagung v.l. Martin Graffenberger, Stefanie Döringer, Robert MusIL, Jakob Eder, Birgit Leick, Josef Lueger, Heike MaYer, Patrick MüLler und Peter GöRGL (Foto: C. Fürthner) 